\title{
Nibaldo Bahamonde Navarro Profesor Emérito, Universidad de Chile
}

\author{
IRMA VILA \& VIVIAN MONTECINO
}

Departamento de Ciencias Ecológicas, Facultad de Ciencias, Universidad de Chile

Por Decreto Exento № 0015396 del 23 de abril de 2004 se otorgó la calidad de Profesor Emérito de la Universidad de Chile a don Jorge Nibaldo Bahamonde Navarro. El 27 de julio se realizó la entrega del diploma en el salón de honor en la Universidad. En esa oportunidad el Rector Prof. Luis Riveros Cornejo señaló "no es este, Nibaldo, un homenaje que se hace para despedir, es, por el contrario, un homenaje que se hace para consolidar una relación de muchos años. Nosotros queremos que nuestros profesores eméritos sigan en la universidad, que estén por siempre en la universidad, porque se trata de su ejemplo, se trata de una vida dedicada con pasión al trabajo universitario, y se trata de retener el valor que eso tiene para las siguientes generaciones".

Este nombramiento es un gran honor para el maestro y para sus discípulos. Por esta razón hemos decidido divulgar la presentación realizada en esta ocasión, que resume la importancia de su aporte, agregando aquí algunos episodios históricos de su vasto quehacer en la academia del país.

\section{CHILOÉ-SANTIAGO-EUROPA-SANTIAGO}

Jorge Nibaldo Bahamonde Navarro nació en 1924 en Ancud, Chiloé, arrullado por los cantos de sirenas, ballenas y barcos fantasmas, los cuales tempranamente inspirarían su vocación por el mar. Cursó sus estudios básicos y secundarios en esa mítica isla, obteniendo su Licencia Secundaria en el Liceo Superior de Ancud en 1939. Su educación universitaria la inició en 1940 en el Instituto Superior de Humanidades de la Facultad de Filosofía y Educación de la Universidad de Chile en Santiago y luego en 1943 ingresó al Instituto Pedagógico de la misma Facultad. A comienzos de 1946 recibió el grado de Licenciado en Filosofía con mención en Ciencias Biológicas y Químicas. Poco tiem- po después obtuvo el título de Profesor de Ciencias Biológicas y Químicas.

En 1951 se casó con Silvia Avilés, quien fue y es un apoyo incondicional para él y nosotros, sus primeras generaciones de estudiantes, tanto en lo humano como en lo académico. La esposa y académica fue la primera profesora de embriología e histología en el Instituto Pedagógico de la Universidad de Chile. Siempre a su lado. Cinco hijos y muchos "ahijados académicos" representan hoy el testimonio de su permanente dedicación.

Becado por la Organización de Naciones Unidas en 1955 y 1956, realizó estudios de postgrado para especializarse en bionomía de especies de importancia económica y en la dinámica de sus poblaciones en institutos de investigación marina y pesquera de Dinamarca, Suecia y Noruega. Durante esta misma estadía se incorporó al Museo Zoológico de la Universidad de Bergen, en Noruega para trabajar con el Prof. H. Brattstroem, con quien analizó las especies del macrobentos recolectados por la expedición de la Universidad de Lund a Chile entre 1948 y 1949, y así facilitó el reconocimiento de especies del contenido gástrico de peces capturados por la expedición en la zona de los canales australes de Chiloé. En el Instituto de Investigaciones Marinas y Pesqueras de Noruega en Bergen participó con G. Rollefsen, G. Saetersdal y F. Devold, en el estudio de la dinámica de poblaciones de bacalao y arenque y se familiarizó con el plancton, oceanografía física y química del mar de Noruega. Visitó otros institutos en Suecia, Noruega, Inglaterra, Francia y Brasil. Viajó expresamente al Museo Nacional de Historia Natural de París para revisar los crustáceos decápodos de Chile descritos por H. Milne Edwards y Nicolet.

Realizó viajes científicos a bordo de varios barcos oceanográficos: el "G. O. Sars”, Noruega, participando en dos cruceros, uno destinado al estudio de la biología del arenque en el mar 
de Noruega, vecindades de Islandia, Isla Faroe y Jan Mayne, y el segundo para el estudio de la biología del bacalao y del "haddock" en las vecindades del mar de Barents e Islas de los Osos. Luego tuvo oportunidad de embarcarse en el "Svén Lovén", en Gullmar Fjord Suecia, en un viaje programado especialmente por el Dr. Gustafson para obtener una visión comparativa entre la fauna bentónica de los canales de Chiloé y los fiordos de Escandinavia. Se embarcó en el "Biologen", barco danés, para recorrer el Little Belt y los fiordos adyacentes, estudiando las relaciones entre las condiciones oceanográficas abióticas y la abundancia de peces.

Su vocación pedagógica destacó tempranamente en su vida académica. En 1943 se inició como ayudante ad honorem en la Cátedra de Biología General del Profesor Parmenio Yáñez, y luego fue ayudante de zoología y jefe de trabajos prácticos en el Departamento de Biología del Instituto Pedagógico de la Facultad de Filosofía y Educación, Universidad de Chile. Entre 1950 y 1956, fue jefe de Laboratorio de Seminarios y Memorias de Cátedra de Zoología del Profesor Guillermo Mann F. Finalmente, en 1957 llegó a ser Profesor del Centro de Investigaciones Zoológicas de la Universidad de Chile para continuar desde 1974 a 1988 como Profesor Titular de la Facultad de Ciencias de esta misma Universidad.

Su docencia de pre y postgrado se extendió a otras escuelas, sedes, colegios regionales y escuelas de temporada de la Universidad de Chile. Incluso encontró tiempo para la docencia en liceos: entre 1947 y 1950 fue Profesor de Ciencias Biológicas en el Liceo Experimental "Darío E. Salas" y desde 1949 a 1950 Asesor Pedagógico, ad honorem del Departamento de Ciencias de la Sección Experimentación Educacional del Ministerio de Educación.

Entre 1950 y 1982 fue jefe de la de Sección Hidrobiología del Museo Nacional de Historia Natural. Desde 1960 colaboró activamente con la carrera de Biología Marina de la Universidad de Chile, con cursos de Carcinología y de Biogeografía Marina, entre otros. En la Estación de Biología Marina en Montemar colaboró en la organización para UNESCO de un curso regional de Entrenamiento de Biología Marina para Latinoamérica. Participó en el curso sobre "Organismos marinos, poblaciones y comunidades del mar", en las Escuelas de Invierno de la Universidad de Chile en Santiago y en provincias.
En los colegios regionales universitarios de La Serena y Talca dictó en 1962 clases de ecología, en 1964 dictó en Valparaíso el curso de Ecología Marina para estudiantes de Biología Marina y entre 1973 y 1974 dictó clases sobre ecología acuática para cursos de ecología general en la Escuela de Medicina de la Sede Norte de la Universidad de Chile. También realizó docencia en las facultades de Agronomía e Ingeniería Forestal y otros centros académicos. Durante 1951 ejerció como Profesor de Zoología y más tarde de Hidrobiología en el Instituto Pedagógico de la Pontificia Universidad Católica de Chile. Participó en actividades de demostración para profesores de ciencias en cursos de verano en la Universidad Técnica Federico Santa María y colaboró también en cursos de ecología para perfeccionamiento de profesores secundarios del Ministerio de Educación.

Un nuevo interés se manifestó conjuntamente en su vida académica: entre 1969 y 1974 inició su perfeccionamiento museológico con seminarios acerca de la organización, distribución y trabajo de los museos gracias a la fructífera colaboración entre la Universidad de Leicester y el Museo Nacional de Historia Natural estimulada y apoyada por UNESCO-ICOM.

\section{DOCENCIA EN HIDROBIOLOGÍA}

En la Universidad de Chile, el curso de Hidrobiología se llamaba en realidad "Zoología Especial". Este era un resquicio a la rígida estructura curricular que permitió abordar nuevos temas y que perseguía interiorizar a los alumnos de Pedagogía en Biología de saberes novedosos como la ecología y en este caso de la ecología de ambientes acuáticos, incluyendo las zonas costeras de El Tabo y Montemar. Junto a Nibaldo, participamos como ayudantes, lo que incluía actividades prácticas sobre la dinámica de poblaciones de la pulga de mar (Emerita analoga) en playas de arena, así como el cálculo de la altura de la marea para poder escalar las rocas y obtener una muestra de la población de chorito maico ( $P e$ rumytilus purpuratus). Cuando las olas daban tregua examinábamos las frondas y aparatos de fijación de los cochayuyos (Durvillea antartica) y de los huiros (Macrocystis). Creemos que este curso estimuló y marcó varias generaciones de profesionales especialmente profesores y licenciados entre 1960 y 1973. 


\section{INVESTIGACIÓN}

Hurgar en el currículum de Nibaldo Bahamonde es embarcarse en un viaje por aguas que corren desde la cordillera al mar, se internan en aguas de la Corriente de Humboldt para fertilizar las aguas costeras desde Arica a los canales magallánicos. Fue precursor de la investigación de recursos pelágicos y bento-demersales del mar chileno incluyendo la descripción de nuevas especies de crustáceos del género Munidopsis (langostinos) y de algunos camarones con trascendencia económica para Chile, como el camarón nailon (Heterocarpus reedi).

Durante mucho tiempo se dedicó al análisis de las tramas tróficas en el océano y al análisis de la biogeografía marina, así como a la biología y taxonomía de crustáceos decápodos (langostas, camarones y centollas). Fue pionero en investigaciones sobre comunidades costeras de las islas oceánicas: San Félix, San Ambrosio, Juan Fernández e Isla de Pascua, obteniendo además las primeras fotografías científicas de la cumbre de San Ambrosio publicadas en textos de geografía de Chile.

Entre sus casi doscientas publicaciones destacan libros, capítulos de libro e informes. Desde el año 1950, describe la alimentación y relaciones tróficas de aves, peces y camarones de ríos chilenos, realiza estudios sobre la fecundidad de camarones y langostinos, estudios sobre poblaciones de pancoras, limanches, y jaibas de importancia comercial en Chile. Más allá del querer saber más, Nibaldo tuvo una manera particular para abordar problemas. Sus preguntas aparentemente simples requerían de gran esfuerzo e información estadísticamente evaluable.

LOS RÍOS MAIPO Y MAPOCHO, LAS PANCORAS, LOS ESTRATIODRILUS, Y OTROS APORTES Y SECRETOS

Fue también una necesidad conocer la fauna de crustáceos límnicos y junto con María Teresa López hubo años de investigación destinados al estudio del ciclo biológico de las pancoras que habitan los sistemas límnicos. El río Maipo, en Talagante, Peñaflor e Isla de Maipo. Realizó recorridos mensuales para registrar la distribución, abundancia y migraciones de estos crustáceos. Cuidadosamente examinamos su morfolo- gía, ciclo de vida y presencia de epibiontes. Entre ellos apareció Stratiodrilus un anélido extraordinariamente importante hasta hoy por sus relaciones biogeográficas.

A los crustáceos era necesario agregar el conocimiento de los peces. Ahora le correspondía a los siguientes estudiantes recorrer el río Maipo nada menos que desde su nacimiento a su desembocadura, utilizando redes y arrastrando chinguillos. Esto representa un registro histórico si se consideran los cambios que ha experimentado este sistema.

En 1990 escribió "Investigaciones marinas como base del desarrollo. Pensamientos desordenados de una experiencia de 50 años". Aquí comparte lo vivido como principal gestor del conocimiento de los recursos costeros del país.

Representante de la Universidad de Chile ante el Comité Oceanográfico Nacional y encargado de la elaboración del Plan Oceanográfico Nacional, como una guía al desarrollo de la investigación marina en el país. Fue presidente de la Comisión de Recursos del Mar y Aguas Continentales del Consejo de Rectores de las Universidades Chilenas. Como uno de los gestores colaboró también en la formulación del Plan Quinquenal de Investigación Científica y Tecnológica Antártica originado en el seminario que para tal propósito se efectuó en 1994.

Su relación con sus discípulos se refleja en cartas que envió y apoyos que no dio: nadie debía pensar que iba a favorecer a alguien. No aceptó funciones directivas aunque formó parte de numerosos comités y participó permanentemente en importantes decisiones con sus ideas e iniciativas científicas. También supimos que el héroe era vulnerable. Nos asustamos cuando sufrió un desmayo en el Hotel O'Higgins, durante una reunión de la Comisión Permanente del Pacífico Sur, de la cual en ese momento era asesor de la delegación chilena.

Como fundador de la Sección de Hidrobiología del Museo Nacional de Historia Natural, donde se desempeñó como jefe durante 32 años, se abocó al estudio de los peces. Congrios, jureles y lenguados fueron examinados por otros estudiantes: Hugo Campos, Germán Pequeño, Julio Movillo, Ismael Kong, Patricio Ojeda y tantos más que hoy ya no están. El Museo nos quitaba su tiempo precioso en la Universidad y solo los días lunes lo teníamos en el Pedagógico (Facultad de Ciencias Natura- 
les) a tiempo completo. Pasaron por este laboratorio los actuales académicos Cecilia Osorio, Carlos Moreno, Eduardo Tarifeño, Carlos Jara, Eduardo Jaramillo, Matilde López y otros como Jaime Caruncho, Guacolda Atria y Eduardo Burlé. La vida académica era intensa, todo se planificaba y se discutía largamente. No era fácil con tantos estudiantes. Promotor persistente de las revistas científicas chilenas y del uso del buen lenguaje español, crítico incansable de los trabajos le valió nuestro apodo de "Tomasito", porque afirmaba "ver y comprobar para creer".

PROGRAMA EL HOMBRE Y LA BIOSFERA

En 1975 se inició el estudio de lagos artificiales. Había que conocer estos sistemas. ¿Cómo se comportarían en el tiempo? Así seguimos a orillas del agua, con más o menos salinidad, levantado piedras, calando redes, estudiando ahora algas y microcrustáceos límnicos en el embalse Rapel. El estudio se completó con el reconocimiento de dos lagos de altura en los Andes y tres en el valle central: Aculeo, Peñuelas y El Plateado. Este proyecto realizado con el apoyo MAB/UNESCO-CONICYT nos cambió el pelaje en términos de financiamiento y equipos (Fig. 1).

Poco tiempo después vino el gran sismo ocasionado por la fusión de dos facultades de ciencias de la Universidad de Chile -fue el año que vivimos en peligro- volvían a repetirse las zozobras de 1973, corría el año 1980. Los cambios sociales de la época lo afectaron muchísimo pero no amilanaron su espíritu de servicio del hombre de ciencias nato que había en él.

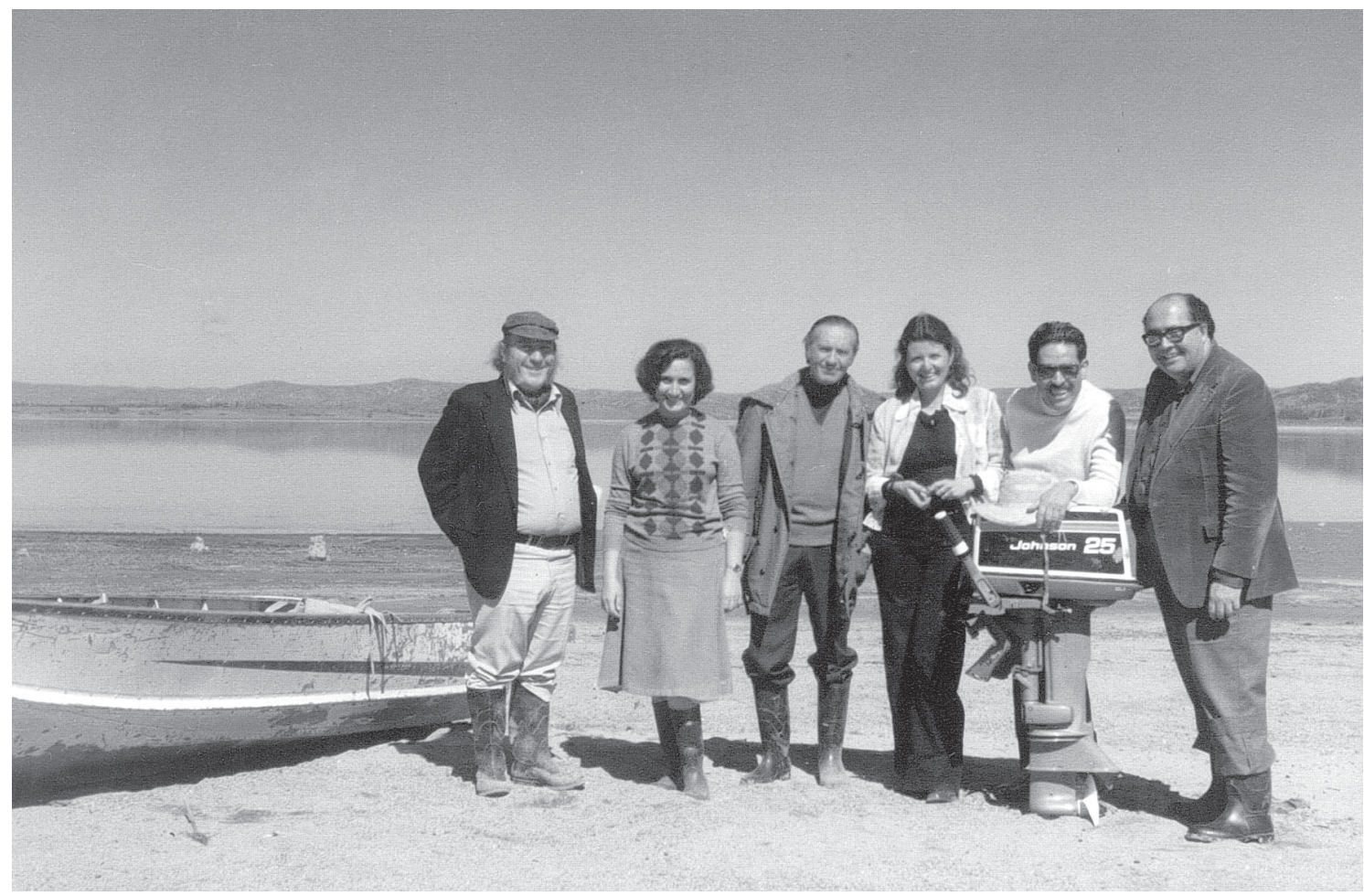

Fig. 1: Visita a los terrenos donados por ENDESA y nueva Estación de Hidrobiología de la Universidad de Chile a orillas del lago Rapel. Nibaldo Bahamonde (segundo de derecha a izquierda) acompañado por Eduardo Bustos, Director del Departamento de Investigación de la Universidad de Chile, Morris Assael de CONICYT, John Celecia de MAB/UNESCO y las autoras de este artículo Irma Vila y Vivian Montecino (fotografía de Sergio Cabrera, 1977).

Visit to the grounds donated by ENDESA and new Hydrobiology Station of the University of Chile at Rapel border. Nibaldo Bahamonde (second from right to left) accompanied by Eduardo Bustos, Director of the Research Department of the University of Chile, Morris Assael, CONICYT, John Celecia MAB/UNESCO and the authors Irma Vila and Vivian Montecino (photo by Sergio Cabrera, 1977). 


\section{RECONOCIMIENTOS}

Su trabajo científico le ha merecido importantes reconocimientos entre los cuales destacamos: a los 26 años de edad, en 1950, recibió el título de "Caballero de la Orden de Vasa" de la Corona Sueca, en reconocimiento por su labor durante la expedición de la Universidad de Lund a Chile, uno de cuyos líderes era el Dr. Hans Brattstroem. En 1954, Miembro Correspondiente de la Sociedad Mexicana de Historia Natural. Entre 1963 y 1973 fue miembro del Scientific Commitee on Antarctic Research (SCAR) y Presidente del Grupo de Biología en Chile. En 1964 recibe la Medalla de la Universidad de San Marcos, Lima, Perú. En 1975 fue galardonado con la Medalla y Diploma de Honor de la Dirección de Bibliotecas, Archivos y Museos por 25 años de labor en la institución. En 1982 obtiene el Diploma de la Sociedad de Biología de Chile y se realiza una Sesión en su Homenaje. En 1984 recibe la Medalla de Oro del Museo Nacional de Historia Natural. Este mismo año se bautiza como "Don Nibaldo" a una embarcación de la Universidad Católica de la Santísima Concepción.

Al conmemorarse los 55 años de la fundación de la Estación de Biología Marina de Montemar (la primera en el Pacífico sureste y cuna de los primeros biólogos marinos del país), la Universidad de Valparaíso lo distingue como Profesor Honorario en 1996. En 1983 es designado Miembro de Número de la Academia de Ciencias del Instituto de Chile y en 1996 recibe el Premio Nacional de Ciencias. En 1997 la Universidad Metropolitana de Ciencias de la Educación lo designó Profesor Emérito.
Un sinnúmero de especies de moluscos, crustáceos, peces e incluso anfibios llevan su nombre, dedicados tanto por sus pares nacionales como internacionales. Destacamos: Batrachila nibaldoi, Projasus bahamondei y Metacrangon bahamondei. Sin embargo, el reconocimiento más importante es agradecerle su personalidad humilde y generosa. Tiempo dedicado a todos, a escuchar pacientemente, y a expresar una respuesta y consejo siempre acertados. El Profesor Bahamonde nos trae los orígenes de la mirada integradora y simple como un libro para aprender a leer, pero al mismo tiempo enciclopédica y estricta como la escuela europea, profunda y contundente. Hizo ciencia con buenas ideas y un mínimo de equipamiento. Enseñó a hacer ciencia por la inquietud de conocer. Sin internet, con calculadoras a rodillo, recorriendo bibliotecas polvorientas, publicando en revistas nacionales. Así, nos ha dejado la importancia del saber y del forjar el conocimiento de los recursos naturales del país, herencia con la cual se ha generado esta filogenia bahamondeana esbozada en 1988 al cumplir 44 años de labor académica y que hoy continúa con los alumnos de sus alumnos (Fig. 2). Grueso y añoso tronco de sólidas raíces que se remontan a Parmenio Yáñez, Ferdinand Oberhauser, Humberto Fuenzalida, Víctor Manuel Baeza, Alfredo Llaña, Luis Capurro, Francisco Riveros y a sus antecesores.

Es nuestro sentir que este árbol continúe generando más y más ramas con hojas frescas que actualicen permanentemente nuestro conocimiento. 


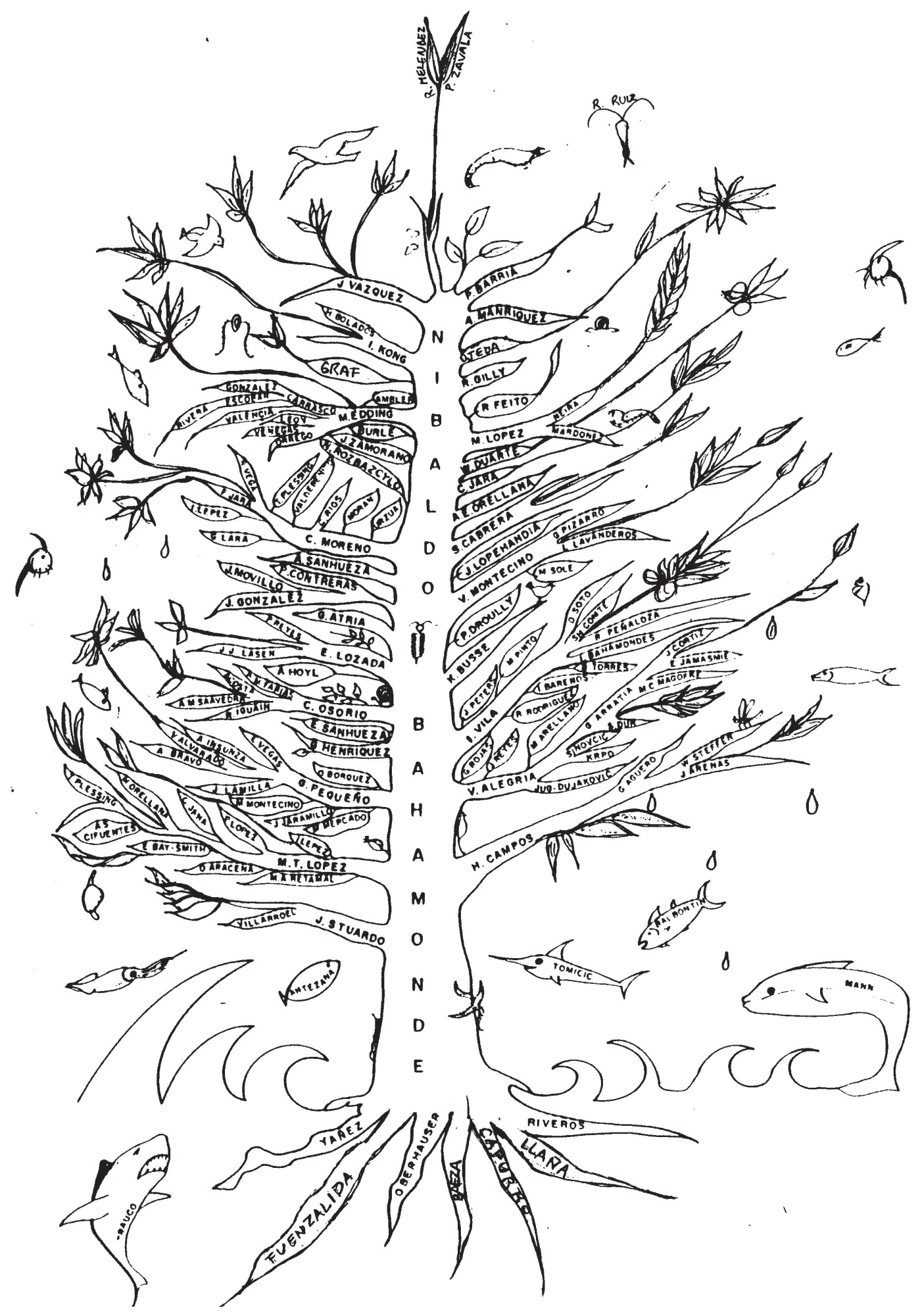

Fig. 2: Árbol genealógico de la "familia académica" del Prof. Nibaldo Bahamonde. Las ramas surgen en todas direcciones y se comunican entre sí, sabemos cuál fue el comienzo pero desconocemos el final.

Genealogical tree of the "academic family" of Prof. Nibaldo Bahamonde. Branches arise in all directions and communicate among them, we know the start but not the end. 